\title{
Determining molecule diffusion coefficients on surfaces from a locally fixed probe: On the analysis of signal fluctuations
}

\author{
Susanne Hahne, ${ }^{1}$ Julian Ikonomov, ${ }^{2}$ Moritz Sokolowski, ${ }^{2}$ and Philipp Maass 1 , 田 \\ ${ }^{1}$ Fachbereich Physik, Universität Osnabrück, Barbarastraße 7, 49076 Osnabrück, Germany \\ ${ }^{2}$ Institut für Physikalische und Theoretische Chemie, \\ Universität Bonn, Wegelerstraße 12, 53115 Bonn, Germany
}

(Dated: December 7, 2012)

\begin{abstract}
Methods of determining surface diffusion coefficients of molecules from signal fluctuations of a locally fixed probe are revisited and refined. Particular emphasis is put on the influence of the molecule's extent. In addition to the formerly introduced autocorrelation method and residence time method, we develop a further method based on the distribution of intervals between successive peaks in the signal. The theoretical findings are applied to STM measurements of copper phthalocyanine $(\mathrm{CuPc})$ molecules on the $\mathrm{Ag}(100)$ surface. We discuss advantages and disadvantages of each method and suggest a combination to obtain accurate results for diffusion coefficients.
\end{abstract}

PACS numbers: 68.43.Jk,68.35.Fx,82.37.Gk

\section{INTRODUCTION}

To describe the growth kinetics of adsorbates on surfaces, knowledge of diffusion coefficients of atoms and molecules on the surface is of vital importance. Different experimental techniques can be utilized to measure corresponding diffusion coefficients. Two of the first methods were Field Emission Microscopy ${ }^{1.2}$ and Field Ionization Microscopy $\stackrel{\underline{3}}{*}$ Another technique is the laser induced thermal desorption,$\frac{4.5}{5}$ where first an area of adparticles is cleared by desorption and then the successive repopulation measured by desorption. For scanning tunneling microscopy (STM) several modes of operation have been described: Taking scans of the surface with the adsorbed particles at different time instants to identify individual particle displacements, $\stackrel{6}{=}$ recording current signals in time with a locally fixed STM tip,$\frac{7}{+}$ and coupling the tip to a single adsorbate and tracking its motions $\underline{\underline{8}}$ Different procedures to extract diffusion coefficients from these and other techniques have been reviewed by Barth in $2000 \stackrel{9}{?}$

Analysis of island densities with predictions from rate equations for submonolayer growth kinetics is a further powerful method, $\stackrel{10,11}{\rightleftharpoons}$ which allows one also to determine binding energies between adatoms or of adatoms to small clusters. An extension of this method to multicomponent systems is given in Refs. 12 and 13 .

In recent years the self-assembly of organic molecules on surfaces has attracted much attention in connection with the idea of developing electronic devices on a molecular level $\stackrel{14}{\underline{14}}$ Molecules in organic surface growth often have high mobilities and sizes large compared to the lattice constant of the substrate. In this situation not all techniques are equally well suited. A convenient and powerful method is the recording of temporal current fluctuations with a fixed scanning tunneling microscope tip $\frac{15}{5}$ or, in principle, of signal fluctuations from any locally fixed microscopic or other probe $\stackrel{2}{2}$ For example, for molecules on insulating substrates, the frequency or height fluctuations in an atomic force microscopy could be used.

In order to extract diffusion coefficients from signal fluctuations of a probe, a theoretical basis for the analysis of the measurement is necessary. One approach was developed for the auto-correlation function (ACF) of an STM signal ${ }^{16,17}$ and applied, on the basis of the corresponding power spectrum, to oxygen atom diffusion on $\mathrm{Si}(111), \frac{15}{\Perp}$ and more recently to hydrogen atom diffusion on $\operatorname{Si}(111) \cdot \frac{18}{n}$ Another approach was followed in Ref. 19 by analyzing the distribution of peak widths in the signal, which correspond to residence times of single molecules under an STM tip. We will refer to it as the residence time distribution (RTD) method in the following. For determining absolute values of diffusion coefficients, a general problem is that the theoretical modeling involves a commonly not precisely known "detection function" with some finite range. However, when the extension of the diffusing molecules is larger than the detection range, this problem becomes essentially irrelevant and the size of the molecule enters as the relevant length scale.

In this work we first revisit both the ACF and the RTD method. For the ACF method we study, as a new aspect, modifications implied by the finite size of molecules and for the RTD method we give a detailed account of the functional form of the RTD in different time regimes. In the RTD, time intervals are considered where the molecule is under (or very close to) the tip. Corresponding time intervals are also included in the calculation of the ACF. It can therefore not be precluded that the interaction of the molecule with the tip is influencing the results. A possible influence can be evaluated systematically by changing the bias voltage, 19 or the distance between tip and substrate. In order to ensure that the influence is small independent of adjustments, we develop a further method, which is based on the analysis of the distribution of peak-to-peak distances in the signal. This method is referred to as the interpeak time distribution (ITD) method in the following.

All three methods are applied to diffusion measurements of copper phthalocyanine $(\mathrm{CuPc})$ on the $\mathrm{Ag}(100)$ 
surface previously reported in Ref. 19. We discuss advantages and disadvantages of the three procedures mentioned above and a possible combination of them, which enables us to improve the quality of results for the diffusion coefficient.

\section{AUTO-CORRELATION FUNCTION (ACF)}

We consider $N$ molecules diffusing on a flat substrate area $A$ and a probing tip of an STM placed at a fixed position above the surface. The lateral tip position is chosen as the origin of an $x$-y-coordinate system on the surface. Each diffusing molecule $\alpha$ with its center at position $\mathbf{r}_{\alpha}(t)=\left(x_{\alpha}(t), y_{\alpha}(t)\right)$ gives a contribution $i_{\alpha}(t)=G\left(\mathbf{r}_{\alpha}(t)\right)$ to the total tunneling current $I(t)=$ $\sum_{\alpha=1}^{N} i_{\alpha}(t)$, where $G(\mathbf{r})$ is the "detection function". We regard the molecules as non-interacting, which should be a valid assumption at low concentrations $c=N / A$, or more precisely on time scales smaller than $l^{2} / D$, where $l \sim c^{-1 / 2}$ is the mean intermolecular distance and $c$ the number density of molecules. The autocorrelation function $C(t)=\langle\delta I(0) \delta I(t)\rangle$ of the current fluctuations $\delta I(t)=I(t)-\langle I\rangle$ can then be written as

$$
\begin{aligned}
C(t) & =N\langle i(0) i(t)\rangle \\
& =c \int \mathrm{d}^{2} \mathbf{r}_{0} \int \mathrm{d}^{2} \mathbf{r}_{1} G\left(\mathbf{r}_{1}\right) f\left(\mathbf{r}_{1}, t \mid \mathbf{r}_{0}\right) G\left(\mathbf{r}_{0}\right),
\end{aligned}
$$

where $f\left(\mathbf{r}_{1}, t \mid \mathbf{r}_{0}\right)$ is the two-dimensional diffusion propagator

$$
f\left(\mathbf{r}_{1}, t \mid \mathbf{r}_{0}\right)=\frac{1}{4 \pi D t} \exp \left(-\frac{\left(\mathbf{r}_{1}-\mathbf{r}_{0}\right)^{2}}{4 D t}\right) .
$$

To provide a quantitative prediction for $C(t)$, knowledge of the detection function $G(\mathbf{r})$ is necessary. For the STM this requires a detailed treatment of the tunneling problem, which is a long-standing problem since the first pioneering work by Tersoff and Hamann $\underline{\underline{20}}$ Based on a simple approach, Sumetskii and Kornyshev $\underline{16}$ suggested a Gaussian detection function for small particles (adatoms) as a result of the tunneling barrier. For extended molecules, the lateral structure of the electronic charge density needs to be taken into account. In fact, without referring to first-principle calculations for the specific system under consideration, it is difficult to obtain accurate expressions for the tunneling current.

While such more detailed treatments are interesting in themselves, they are not really required for determining the diffusion coefficient $D$. A more practical approach is to transform $I(t)$ into a rectangular signal $I_{\text {rec }}(t)=\Theta\left(I(t)-I_{c}\right)$, where $\Theta($.$) is the Heaviside jump$ function $\left(\Theta(x)=1\right.$ for $x>0$ and zero else), and $I_{c}$ is a threshold current to exclude background noise. If we consider situations, where at most one molecule can be in the detection region under the tip, each single molecule contribution $i_{\alpha}(t)$ to $I_{\text {rec }}(t)$ also transforms to a rectangular signal $i_{\alpha}^{\mathrm{rec}}(t) \in\{0,1\}$, and $I_{\text {rec }}(t)=\sum_{\alpha} i_{\alpha}^{\mathrm{rec}}(t) \in\{0,1\}$.
Accordingly, the detection function can be defined as "indicator function" of the molecule

$$
G(\mathbf{r})= \begin{cases}1 & \text { if } \mathbf{r} \in \mathcal{M} \\ 0 & \text { else }\end{cases}
$$

where $\mathcal{M}$ is the set of center positions of the molecule which give rise to a tunneling current $I>I_{c}$. To get a description that is independent of details of the molecule's shape, we assign a circle to the set $\mathcal{M}$. The radius $R$ of this circle can, for example, be determined by setting the covered area of the molecule equal to $\pi R^{2}$, by the gyration radius of the set $\mathcal{M}$, or by some other reasonable requirement. The function $G(\mathbf{r})$ from Eq. (3) is then approximated by $\Theta(R-|\mathbf{r}|)$.

The mutual exclusion under the tip implies that the molecules are no longer non-interacting as assumed when deriving Eq. (11). This leads to nonzero cross-correlations $\left\langle i_{\alpha}^{\mathrm{rec}}(t) i_{\beta}^{\mathrm{rec}}(0)\right\rangle, \alpha \neq \beta$. While these cross-correlations could be treated approximately, we concentrate here on times smaller than the mean residence time $\sim \tau_{R}:=$ $R^{2} / D$ of a molecule under the tip. In this regime we can set $\left\langle i_{\alpha}^{\mathrm{rec}}(t) i_{\beta}^{\mathrm{rec}}(0)\right\rangle \simeq 0$ for $\alpha \neq \beta$. Accordingly, the self-part $N\left\langle i_{\alpha}^{\mathrm{rec}}(t) i_{\alpha}^{\mathrm{rec}}(0)\right\rangle$ appearing in Eq. (1) gives the correlation function $C_{\text {rec }}(t)=\left\langle I_{\text {rec }}(t) I_{\text {rec }}(0)\right\rangle$ (i.e. without subtracting a term $\left.\left\langle I_{\text {rec }}\right\rangle^{2}\right)$. Evaluation of Eq. (1) then yields

$$
\begin{aligned}
C_{\text {rec }}(t)=4 \pi c \int_{0}^{R} \mathrm{~d} r_{0} & r_{0} \int_{0}^{R} \mathrm{~d} r_{1} r_{1} \\
\times & \frac{\exp \left(-\frac{r_{0}^{2}+r_{1}^{2}}{4 D t}\right)}{4 D t} I_{0}\left(\frac{2 r_{0} r_{1}}{4 D t}\right),
\end{aligned}
$$

where $I_{0}$ is the modified Bessel function of zeroth order.

The knowledge of $C_{\text {rec }}(t)$ for times $t<\tau_{R}$, as predicted by Eq. (4) for single-particle diffusion, is sufficient to determine $D$. For $t>\tau_{R}$, collective properties and the associated cross-correlations had to be taken into account. In a rough treatment one could factorize $\left\langle i_{\alpha}^{\mathrm{rec}}(t) i_{\beta}^{\mathrm{rec}}(0)\right\rangle \simeq\left\langle i_{\alpha}^{\mathrm{rec}}(t)\right\rangle\left\langle i_{\beta}^{\mathrm{rec}}(0)\right\rangle$, which amounts to add $\left(c \pi R^{2}\right)^{2}$ to the expression in Eq. (4) for $t \gg \tau_{R}$.

\section{Application to $\mathrm{CuPc}$ on $\mathrm{Ag}(\mathbf{1 0 0})$}

A relaxed structure of the $\mathrm{CuPc}$ molecule in vacuum after energy minimization, as obtained from a DFT calculation, is displayed in Fig. 1, together with. A circle of radius $R=7.6 \AA$ is assigned to it. The relaxed structure can be expected to be essentially unmodified when the $\mathrm{CuPc}$ molecule is adsorbed on the $\mathrm{Ag}(100)$ surface. STM studies combined with DFT calculations showed that transition metal phtalocyanines generally lie flat on the $\mathrm{Ag}(100)$ surface $^{21}$ This is in agreement with STM images of CuPc islands on $\mathrm{Ag}(100) \underline{22}$

After deposition of CuPc molecules on $\mathrm{Ag}(100)$ up to a coverage of $10-15 \%$, islands form and a quasi-stationary state is reached, where the rates of detachment from and 


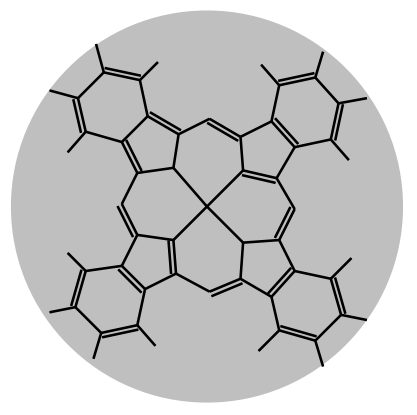

FIG. 1. Relaxed structure of the CuPc molecule. Notice that the terminating bonds are connected to hydrogen atoms. The assigned detection area marked in gray is a circle with radius $R=7.6 \AA$. It corresponds to the smallest circle that entails all nuclei.

attachment to islands balance each other. In this quasistationary state the diffusing $\mathrm{CuPc}$ molecules have an effective small number density $c$. Then the tunneling current was measured for a stationary STM tip and constant bias voltage. The height of the tip was preset indirectly by the choice of setpoint current $I_{\text {setpoint }}$ and bias voltage $U_{\text {bias. }}$. Further details of the experiment are given in Ref. 19.

The diffusing molecules cause fluctuations in the tunneling current $I(t)$ as shown in Fig. 22 (a) for a time interval of $2 \mathrm{~ms}$ at $T=192 \mathrm{~K}$. A histogram of the tunnelling current, determined for the whole time series of

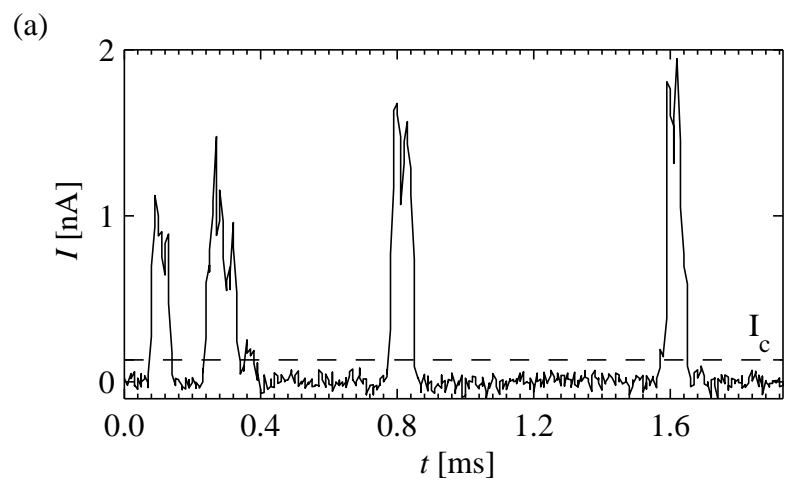

(b)

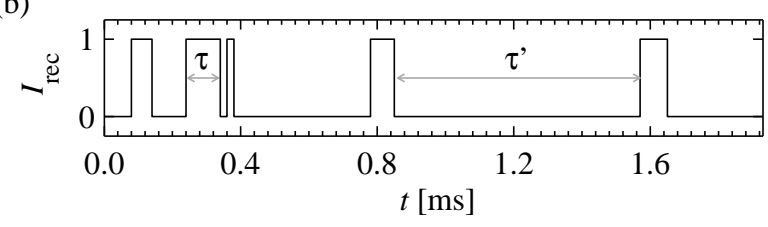

FIG. 2. (a) Fluctuating STM tunneling current $I(t)$ observed during $\mathrm{CuPc}$ diffusion on $\mathrm{Ag}(100)$ at $T=192 \mathrm{~K}\left(U_{\text {bias }}=1.7 \mathrm{~V}\right.$, $\left.I_{\text {setpoint }}=20 \mathrm{pA}\right)$. The dashed line marks the threshold value $I_{c}$, above which the distribution of the current values has no longer a Gaussian shape, see Fig. 3. (b) The rectangular signal $I_{\text {rec }}(t)=\Theta\left(I(t)-I_{c}\right)$ associated with $I(t)$. The widths of the rectangles give the residence times $\tau$ and the distances between the rectangles give the interpeak times $\tau^{\prime}$.

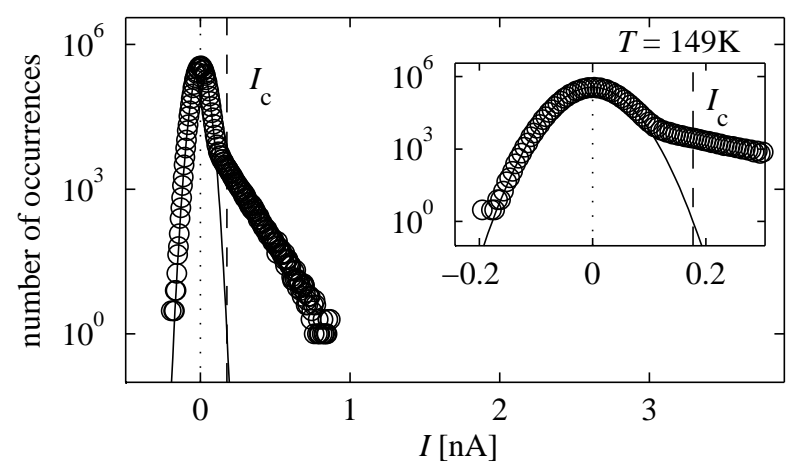

FIG. 3. Histogram of measured current values for $T=192 \mathrm{~K}$ after shifting the maximum to $I=0$. The solid line marks a Gaussian fit with zero mean and standard deviation determined from the histogram for negative currents. The inset shows an enlargement to demonstrate the determination of the threshold value $I_{c}$ used for separating the diffusioninduced signal from the noise (see text).

length $40 \mathrm{~s}$, is depicted in Fig. 3, In this figure, the maximum of the histogram was shifted to $I=0 \underline{\underline{23}}$ Negative $I$ values are associated with noise and their distribution can be fitted to a half-sided Gaussian. Extending this Gaussian distribution to positive $I$ values yields the solid line in Fig. 3. For small positive $I$ values this curve fits very well the data, implying that these values can also be attributed to noise. The diffusing $\mathrm{CuPc}$ molecules cause deviations from the Gaussian distribution at larger $I$ values. To separate the diffusion-induced fluctuations from the noise, we define a threshold value $I_{c}>0$, where the Gaussian histogram of the number of occurrences drops below one, cf. Fig. 3. This threshold is marked by the dashed line in Fig. 2(a) and the corresponding $I_{\text {rec }}(t)$ is shown in Fig. 2(b). Using this procedure we determined the ACF of $I_{\text {rec }}(t)$ for seven different temperatures investigated in the experiments $\underline{19}$

Normalized ACFs $C_{\text {rec }}(t) / C_{\text {rec }}(0)$ for two temperatures are shown in Fig. 4(a) (symbols) and fits to them with Eq. (4) and under the constraint $t<\tau_{R}$ are marked as solid lines. The resulting diffusion coefficients are displayed in the Arrhenius plot in Fig. 4(b) (symbols), where a least square fit of $D=D_{0} \exp \left(-E_{a} / k_{\mathrm{B}} T\right)$ to these data (solid line) yields an activation energy $E_{a}=30 \mathrm{meV}$ and a pre-exponential factor $D_{0}=10^{-9.4} \mathrm{~cm}^{2} / \mathrm{s}$.

\section{RESIDENCE TIME DISTRIBUTION (RTD)}

The residence times $\tau$ are the time intervals between entrance and exit of a molecule into the detection region $\mathcal{M}$ of the probe, see Fig. 2. By sampling these $\tau$ values, the $\operatorname{RTD} \Psi(\tau)$ is obtained.

For a theoretical description of the RTD we need to tackle the problem of the diffusion of a molecule center in a circle with radius $R$ and absorbing boundaries, see Fig. 5a . Since the molecule center cannot be placed at 
(a)

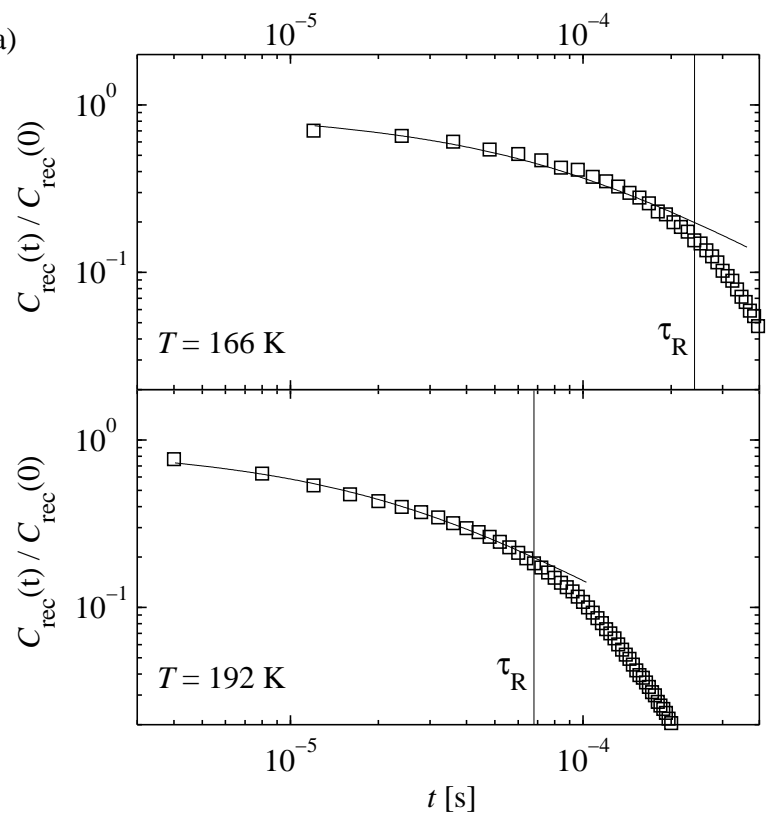

(b)

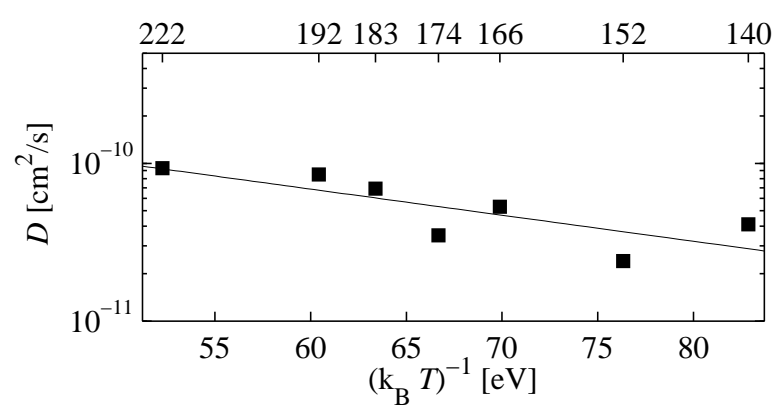

FIG. 4. (a) Normalized ACFs $C_{\mathrm{rec}}(t) / C_{\mathrm{rec}}(0)$ as a function of time for two temperatures (symbols) and best fits with Eq. (4) for $t<\tau_{R}$ (solid lines). (b) Arrhenius plot of the resulting diffusion coefficients (symbols). A least square fit (solid lines) according to the Arrhenius law yield $E_{a}=30 \mathrm{meV}$ and $D_{0}=$ $10^{-9.4} \mathrm{~cm}^{2} / \mathrm{s}$.

the absorbing boundary, its initial center position is considered to be at a distance $\epsilon R$ from the boundary. This means that the initial probability density of the center position is a delta-function concentrated on the ring with radius $(1-\epsilon) R$. Physically the length $\epsilon R$ may be associated with an elementary step size of the molecule on the substrate.

Due to the symmetric initial condition, the probability density for the molecule center position $\mathbf{r}$ at time $t$ depends on $r=|\mathbf{r}|$ only and is given by (see Appendix)

$$
p(\mathbf{r}, t)=\sum_{n=1}^{\infty} \frac{J_{0}\left(\alpha_{n} r / R\right)}{\pi R^{2}} \frac{J_{0}\left(\alpha_{n}(1-\epsilon)\right)}{J_{1}^{2}\left(\alpha_{n}\right)} e^{-\alpha_{n}^{2} t / \tau_{R}}
$$

where $J_{k}($.$) are the Bessel functions of order k$, and the $\alpha_{n}$ are the (positive) zeros of $J_{0}, J_{0}\left(\alpha_{n}\right)=0$ with $0<$ $\alpha_{1}<\alpha_{2}<\ldots$ (a)

(b)
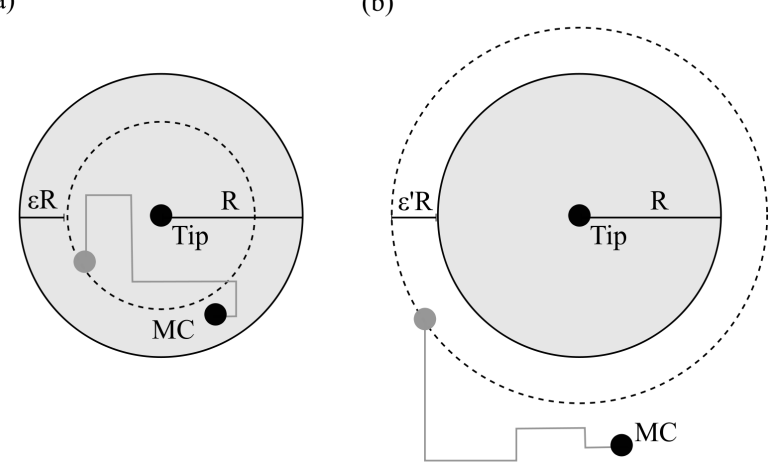

FIG. 5. Illustration of the geometry used for the calculation of (a) the RTD and (b) the ITD. The dashed lines indicate the uniformly distributed initial probabilities on the rings displaced by $\epsilon R$ (a) and $\left(\epsilon^{\prime} R\right)$ (b) from the absorbing boundary (solid lines). MC stands for the diffusing molecule center.

With $p(\mathbf{r}, t)$ from Eq. (5), the calculation of the RTD amounts to calculating the first passage time distribution with respect to the absorbing boundary. This can be carried out by applying standard techniques from random walk theory, as described, for example, in Ref. 24 . The probability that the molecule center has not left the circle until time $t$ is $\phi(t)=2 \pi \int_{0}^{R} \mathrm{~d} r \operatorname{rp}(\mathbf{r}, t)$. The probability that the center leaves the circle in the time interval $[\tau, \tau+\Delta \tau]$ is $\phi(\tau)-\phi(\tau+\Delta \tau)$, implying that the probability density $\psi(\tau)$ for the residence times $\tau$ is $\psi(\tau)=-2 \pi \partial_{\tau} \int_{0}^{R} \mathrm{~d} r \operatorname{rp}(\mathbf{r}, \tau)$. This yields

$$
\psi(\tau)=\frac{2}{\tau_{R}} \sum_{n=1}^{\infty} \alpha_{n} \frac{J_{0}\left(\alpha_{n}(1-\epsilon)\right)}{J_{1}\left(\alpha_{n}\right)} \mathrm{e}^{-\alpha_{n}^{2} \tau / \tau_{R}} .
$$

We note that the characteristic time $(\epsilon R)^{2} / D$ sets a lower limit above which Eq. (5) becomes applicable, because one would have to refine the continuum treatment for smaller $\tau$. For the experiments analyzed here, this is not of relevance because such small $\tau$ are not resolved.

For $\tau \gtrsim(\epsilon R)^{2} / D$ the functional form of the solution changes with respect to the characteristic time $\tau_{R}$ the molecule needs to explore the circle area. For $\tau \ll \tau_{R}$, we find

$$
\psi(\tau) \propto \frac{1}{\tau_{R}}\left(\frac{\tau}{\tau_{R}}\right)^{-3 / 2} .
$$

This power law can be understood by considering the (negative) time derivative of the probability that the molecule is next to the absorbing boundary at time $t$ [i.e. the efflux rate, which equals $\psi(\tau)]$. For $\tau \ll \tau_{R}$ this probability is given by the ratio of the explored boundary section $(\propto \sqrt{\tau})$ to the explored area inside the circle $(\propto \tau)$, yielding $\Psi(\tau) \sim-\partial_{\tau} \sqrt{\tau} / \tau \sim \tau^{-3 / 2}$.

For $\tau \gg \tau_{R}$, we find

$$
\psi(\tau) \sim \frac{2}{\tau_{R}} \frac{\alpha_{1} J_{0}\left(\alpha_{1}(1-\epsilon)\right)}{J_{1}\left(\alpha_{1}\right)} \mathrm{e}^{-\alpha_{1}^{2} \tau / \tau_{R}} .
$$


This result can be interpreted by noting that for $\tau \gg$ $\tau_{R}$, where the occupation probability of the molecule is spread over the circle, the efflux rate is essentially constant and given by the inverse of the time $\tau_{R}$ for a molecule to reach the boundary. A Poisson process with this constant rate yields $\Psi(\tau) \sim \tau_{R}^{-1} \exp \left(-\right.$ const. $\left.\tau / \tau_{R}\right)$

\section{Application to measurements}

For the diffusion of $\mathrm{CuPc}$ on $\mathrm{Ag}(100)$ the $\tau$ values were extracted from the rectangular current signal as described in Fig. 2. Distributions $\Psi(\tau)$ of these $\tau$ values are shown in Fig. 6(a) for two representative temperatures. By fitting the exponential tail with Eq. (8), we obtain the diffusion coefficient $D$. In a self-consistency check we have assured that the tail regime used for the fitting fulfills the requirement $\tau \gg \tau_{R}=R^{2} / D$. In addition the value $\epsilon$ can be calculated from the prefactor of the fit. We find $\epsilon \simeq 0.3$, which means that $\epsilon R$ is about the lattice constant $a=2.89 \AA$ of the $\operatorname{Ag}(100)$ substrate. Inserting $D$ and $\epsilon$, Eq. (6) yields the full distribution $\psi(\tau)$, which is marked by the solid lines in Fig. 6(a). The very good agreement of the theoretical prediction with the measured data demonstrates the reliability of the approach.

The diffusion coefficient $D$ is shown in Fig. 6(b) for the two temperatures in (a) together with the five further investigated temperatures in an Arrhenius plot. From the slope of the fitted line, we find an activation energy $E_{a}=$ $30 \mathrm{meV}$ and a pre-exponential factor $D_{0}=10^{-9.4} \mathrm{~cm}^{2} / \mathrm{s}$. These values agree with the ones of the ACF analysis.

We note, however, that these values deviate from those reported in Ref. $19\left(E_{a}=81 \mathrm{meV}\right.$ and $D_{0}=$ $10^{-8.4} \mathrm{~cm}^{2} / \mathrm{s}$ ). The difference is due to the fact that error bars were taken into account in the analysis performed in Ref. 19. One point in the Arrhenius plot, which was determined from the data set measured for $T=222 \mathrm{~K}$, had a particularly small error and was largely influencing the slope of the fit line because of its exposed position with respect to the other points. When excluding this particular point from the fitting, values $E_{a}=38 \mathrm{meV}$ and $D_{0}=10^{-9.7} \mathrm{~cm}^{2} / \mathrm{s}$ are obtained, in fair agreement with the present analysis. We have refrained from including error bars here because the fits of the RTD for different temperatures yielded comparable errors and small differences between them seem to be insignificant with respect to other possible sources of errors, as, for example, minor temperature fluctuations or the influence of spatial inhomogeneities in diffusion profiles, that are associated with the fact that islands act as sinks for the molecule diffusion.

\section{INTER-PEAK TIME DISTRIBUTION (ITD)}

The inter-peak times $\tau^{\prime}$ are the time intervals between the end of a peak and the beginning of the next peak in (a)
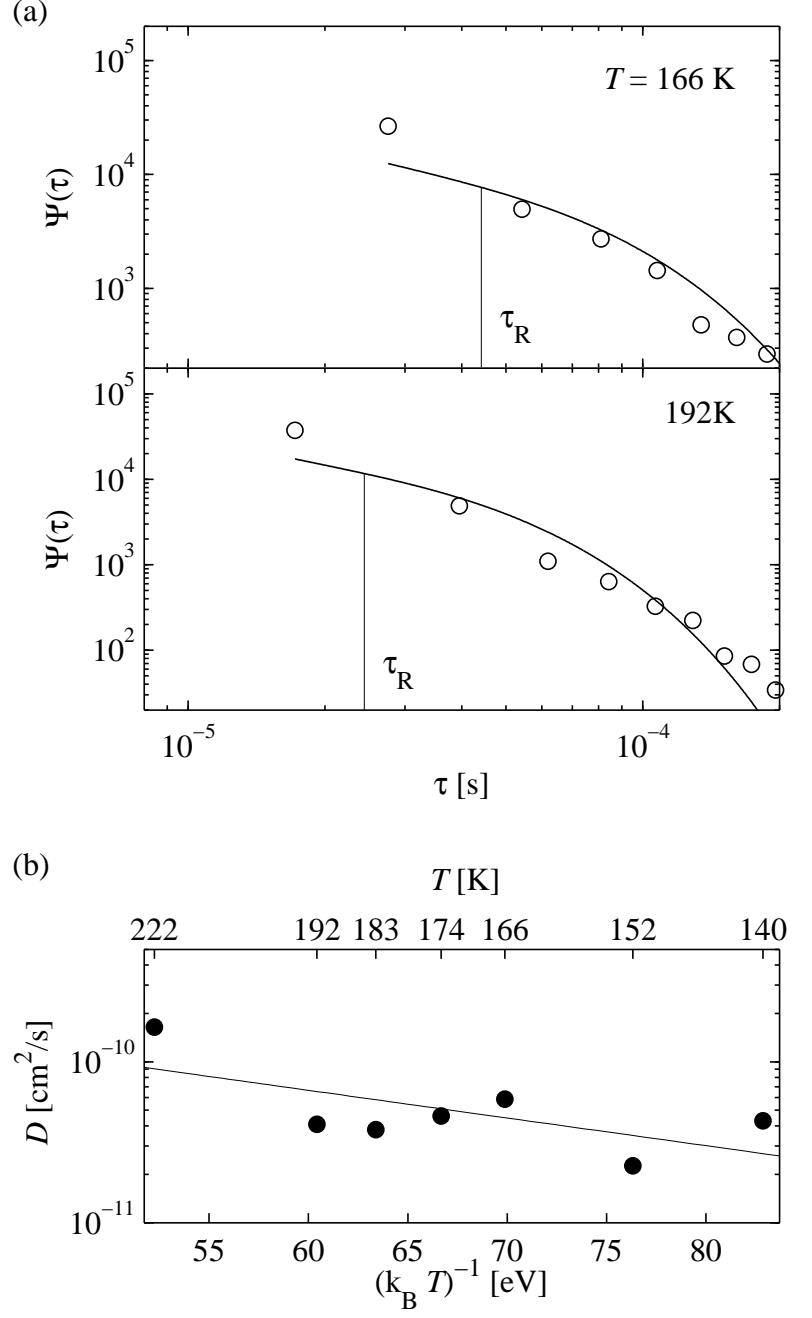

FIG. 6. (a) Residence time distribution for $\mathrm{CuPc} / \mathrm{Ag}(100)$ at two temperatures (symbols). The solid line marks the result predicted by Eq. (5) after determining the parameters $D$ and $\epsilon$ from the exponential tail region $\tau \gg \tau_{R}$ (cf. Eq. (8) and discussion in text). (b) Arrhenius plot of the extracted diffusion coefficients. A least square fit (solid line) with the Arrhenius law yields an activation energy $E_{a}=30 \mathrm{meV}$ and a pre-exponential factor $D_{0}=10^{-9.4} \mathrm{~cm}^{2} / \mathrm{s}$.

the rectangular signal, see Fig. 22 The statistics of them is, for small $\tau^{\prime}$, dominated by entrance and exit of the same molecule into the detection region $\mathcal{M}$. For calculating the contribution of these return processes to the ITD we analyze the diffusion of a molecule center with initial distance $\epsilon^{\prime} R$ from a circular absorbing boundary with radius $R$, see Fig. [5. For the probability density of the molecule center to be at position $\mathbf{r}$ at time $t$ one obtains (see Appendix)

$$
\begin{aligned}
p(\mathbf{r}, t)=\int_{0}^{\infty} \frac{\mathrm{d} \alpha}{2 \pi R^{2}} & \alpha W_{0}(\alpha r / R, \alpha) \\
& \times \frac{W_{0}\left(\alpha\left(1+\epsilon^{\prime}\right), \alpha\right)}{J_{0}^{2}(\alpha)+Y_{0}^{2}(\alpha)} \mathrm{e}^{-\alpha^{2} t / \tau_{R}},
\end{aligned}
$$


where $W_{0}(x, y)=J_{0}(x) Y_{0}(y)-J_{0}(y) Y_{0}(x)$ and $Y_{0}$ is the zeroth order Bessel function of second kind.

The ITD can be derived analogously to the treatment of the RTD by taking the time derivative of the integral of $p(\mathbf{r}, t)$ over the outer area with respect to the circle. In the present case it is more convenient to take the flow through the absorbing boundary, $\psi\left(\tau^{\prime}\right)=$ $\oint \mathrm{d} \mathbf{s} \cdot[-D \nabla p(\mathbf{r}, t)]_{r=R}$, which, when making use of the Wronskian $\left[J_{0}^{\prime}(x) Y_{0}(x)-J_{0}(x) Y_{0}^{\prime}(x)\right]=2 / \pi x$, yields

$$
\psi\left(\tau^{\prime}\right)=\frac{2}{\pi \tau_{R}} \int_{0}^{\infty} \mathrm{d} \alpha \alpha \frac{W_{0}\left(\alpha\left(1+\epsilon^{\prime}\right), \alpha\right)}{J_{0}^{2}(\alpha)+Y_{0}^{2}(\alpha)} \mathrm{e}^{-\alpha^{2} \tau^{\prime} / \tau_{R}} .
$$

For $\tau^{\prime} \ll \tau_{R}$, the asymptotic behavior for $\tau^{\prime} \rightarrow 0$ is

$$
\psi\left(\tau^{\prime}\right) \sim \frac{\epsilon^{\prime}}{2 \tau_{R} \sqrt{\pi\left(1+\epsilon^{\prime}\right)}} \exp \left(-\frac{\epsilon^{\prime 2} \tau_{R}}{4 \tau^{\prime}}\right)\left(\frac{\tau^{\prime}}{\tau_{R}}\right)^{-3 / 2} .
$$

Accordingly, $\psi\left(\tau^{\prime}\right)$ rapidly rises for small $\tau^{\prime}$ and, after going through a maximum $\psi_{\max }$ at $\tau_{\max }^{\prime}=\left(\epsilon^{\prime} R\right)^{2} / 6 D$, approaches a power law with exponent $(-3 / 2)$. This power law has an analogous physical origin as the power law in the RTD, see the discussion in Sec. [II after Eq. (7). Here $\tau_{R}$ is the typical time where the molecule center in Fig. 5 realizes the finite extent of the detection area, or, in other words, where the molecules realizes its size.

For $\tau^{\prime} \gg \tau_{R}$, Eq. (10) can be approximated by

$$
\psi\left(\tau^{\prime}\right) \simeq \frac{2 \ln \left(1+\epsilon^{\prime}\right)}{\tau^{\prime} \ln ^{2}\left(\tau^{\prime} / \tau_{R}\right)} .
$$

The asymptotics $\sim\left(\tau \ln ^{2} \tau\right)^{-1}$ follows from the fact that for large $\tau^{\prime}$ the detection area becomes very small with respect to the area explored by the molecule. Accordingly, $\Psi\left(\tau^{\prime}\right)$ scales as the probability of first return to the origin of a two-dimensional random walk.

The large $\tau$ behavior predicted by Eq. (12) is, however, of limited use, because another molecule can enter the detection area before the molecule, which has left this area at last, returns to it. The memory to a molecule that leaves the detection area is lost on time scales of order $l^{2} / D \sim 1 / c D$. On these time scales different molecules can be regarded as entering the detection area with a constant rate. This rate should scale with the inverse mean time $D / l^{2}$ for a molecule outside the detection area to enter it. Hence in the limit of large $\tau^{\prime}$, an exponential distribution is expected,

$$
\psi\left(\tau^{\prime}\right) \sim c D \exp \left(-\kappa \pi D c \tau^{\prime}\right),
$$

where $\kappa$ is a constant of order unity.

\section{Application to measurements}

The inter-peak intervals were sampled from the current signal as explained in Fig. 2 and the ITDs for two temperatures are shown in Fig. 7(a). Note that for very
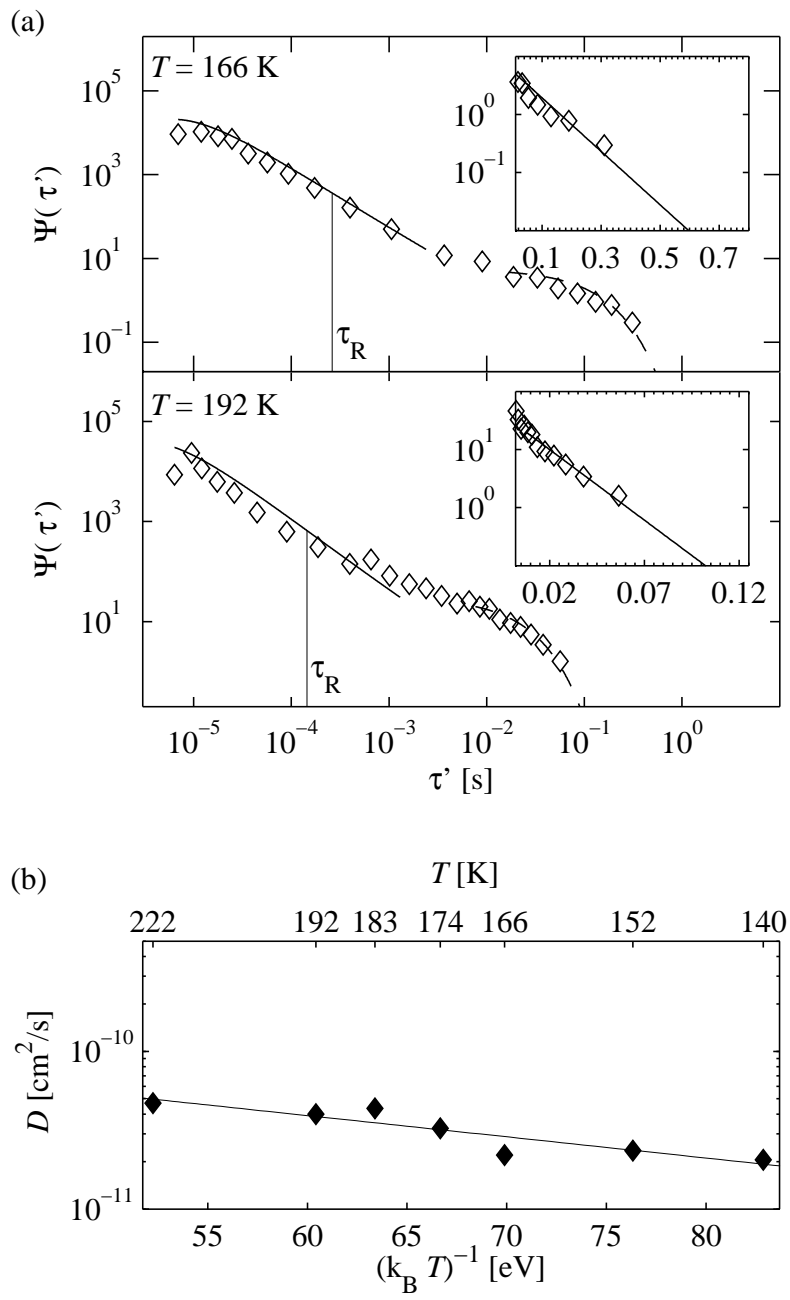

FIG. 7. (a) ITD for $\mathrm{CuPc} / \mathrm{Ag}(100)$ for two temperatures. The inset shows the exponential decay at large times, where the straight solid lines (dashed lines in the main log-log plots) are fits with Eq. (13), yielding $D$ (see text). Using these $D$ values, the solid lines in the main plots mark the short time behavior after fitting with Eq. (11). (b) Arrhenius plot of the extracted diffusion coefficients for all seven investigated temperatures. A least square fit (solid line) with the Arrhenius law yields an activation energy $E_{a}=31 \mathrm{meV}$ and a pre-exponential factor $D_{0}=10^{-9.6} \mathrm{~cm}^{2} / \mathrm{s}$.

small times the data indicate a plateau, which is not contained in Eq. (11). A refinement of the continuum description would be necessary to capture this behavior, analogous to the very small times in the RTD. For times $\tau \gtrsim \tau_{R}$ the power law predicted by Eq. (11) is reflected by the straight line behavior in the double-logarithmic plot, before eventually the exponential decay according to Eq. (13) takes over.

To obtain $D$ from the ITD, we first concentrate on the long-time behavior. Setting $\kappa=1$, the rate $c D$ of the exponential decay is provided by the slopes of the lines shown in the insets of Fig. 7(a). The concentration is determined from the probability $c \pi R^{2}=\sum_{i} \tau_{i} / T$ for a 
TABLE I. Strengths and weaknesses of the three methods for determining $D$.

\begin{tabular}{llllll}
\hline \hline Characteristics & ACF & RTD & ITD \\
\hline \hline Signal processing & $\oplus$ & Convenient by FFT & $\ominus$ & $\begin{array}{l}\text { Peak widths affected by } I_{c} \\
\text { value }\end{array}$ & $\begin{array}{l}\text { Negligible influence of } I_{c} \\
\text { on interpeak distances }\end{array}$ \\
\hline Tip influence & $\ominus$ & Possible & $\ominus$ & Possible & Less likely \\
\hline Assumptions & $\ominus$ & Non-interacting particles & $\oplus$ & None & $\begin{array}{l}\text { None in short-time regime } \\
\text { Non-interacting } \\
\end{array}$ \\
& & & & $\begin{array}{l}\text { particles } \\
\text { in long-time regime }\end{array}$ \\
\hline \hline
\end{tabular}

molecule to be in the detection area, where $T=\sum_{i}\left(\tau_{i}+\right.$ $\left.\tau_{i}^{\prime}\right)$ is the total measurement time. For the seven data sets taken at different temperatures, we find $c$ values in the range $0.6-5 \times 10^{-6} \AA^{2}$, corresponding to coverages $\Theta=0.01-0.08 \%$ of the diffusing $\mathrm{CuPc}$ molecules. The resulting $D$ values are shown in the Arrhenius plot in Fig. 7(b) and yield an activation energy of $E_{a}=31 \mathrm{meV}$ and a pre-exponential factor of $D_{0}=10^{-9.6} \mathrm{~cm}^{2} / \mathrm{s}$. Note that the assumed value $\kappa=1$ affects only the prefactor $D_{0}$ but not the activation energy $E_{a}$.

With $D$ obtained from the long-time behavior, we can fit the remaining part of the ITD with Eq. (11). Corresponding curves are shown as solid lines in the doublelogarithmic plots of Fig. 7(a). They give a good agreement with the experimental data. The fits yield $\epsilon^{\prime} \simeq 0.5$, which is consistent with $\epsilon \simeq 0.3$ obtained in the analysis of the RTD, see Sec. III. Accordingly, we find again that $\epsilon^{\prime} R$ is of the order of the lattice constant $a$, as one should expect.

In principle, the part of the ITD dominated by the single molecule diffusion can also be used to determine $D$. For this we have to notice that the necessary refinements of the continuum theory to describe the behavior of the ITD left to the maximum

$$
\psi_{\max }=\frac{3 \sqrt{6} D}{\sqrt{\pi\left(1+\epsilon^{\prime}\right) \mathrm{e}^{3 / 2}\left(\epsilon^{\prime} R\right)^{2}}} \approx \frac{D}{a^{2}} .
$$

are not expected to yield larger values of the ITD. In fact, when considering jump dynamics of the molecules with a rate $D / a^{2}$ for short times, the ITD should behave as $\sim\left(D / a^{2}\right) \exp \left(-\right.$ const. $\left.D \tau^{\prime} / a^{2}\right)$, i.e. the largest value of the ITD should be of order $D / a^{2}$. Due to matching with the continuum treatment, we can identify $\psi_{\max }$ with the maxima seen in Fig 7(a). This then is a convenient way to determine $D /\left(\epsilon^{\prime} R\right)^{2}$, and knowing this value, to extract $D$ by fitting the part right to the maximum predicted by Eq. (11). Application of this alternative method indeed yields values for $D$ and $\epsilon^{\prime}$ in good agreement with those discussed above.

\section{COMPARISON OF THE METHODS}

For applications it is important to clarify how the three methods are best combined to obtain most accurate results for $D$. To this end we need to evaluate the strengths and weaknesses of each method. Let us first note that all methods work with a single input parameter, which is the molecule radius $R$. All other variables arise from the analyses described in Secs. II]IV] Because $\tau_{R}=R^{2} / D$ is actually determined, uncertainties with respect to $R$ slightly affect the diffusion coefficient.

Table $\llbracket$ summarizes the advantages and disadvantages of the three methods. The ACF can be readily calculated by a fast Fourier transformation without caring about peak identification in the signal. A disadvantage is that only the short-time regime $t \lesssim \tau_{R}$ is governed by single particle diffusion, while an accurate theoretical description of the crossover to the long-time regime, governed by collective particle diffusion, requires a careful consideration of the mutual exclusion of the molecules (and possibly other interaction effects). The decay of the correlation function within this regime can be small, which then affects the accuracy of the $D$ values resulting from the fitting. Another drawback is that the determination of the ACF includes time intervals where molecules are under the tip and possible interactions with the tip can thus have an influence on the diffusion properties.

Both the RTD and ITD method require some more effort in preparation of the data, which is connected to the determination of the threshold current $I_{c}$ and identification of the peaks. Once $I_{c}$ is set, both the peak widths and interpeak distances can be extracted simultaneously. Note that any method of determining $I_{c}$ is associated with some uncertainty. For the ITD this is no problem in practice, because the interpeak intervals are comparatively large. We have checked that, when taking the interpeak distances as time intervals between peak maxima, the results do not change significantly. For the RTD the uncertainty of $I_{c}$ is a more severe problem. Because molecules diffuse slowly into the detection area, the peaks in the original tunneling current signal have rather flat flanks. As a result the peak widths change more sensitively with $I_{c}$ than the interpeak intervals.

An advantage of the RTD is that $D$ can be determined solely by analyzing the exponential tail for large residence times. One should note, however, that it may be difficult to obtain a good statistics in the tail regime, when the molecules are highly mobile or small. In this case the peaks are narrow and it could be difficult to resolve them accurately. In the RTD method the interaction with the tip can influence the residence times and in this 
case one would not determine the free diffusion of the molecules on the substrate. By systematically changing the bias voltage, a possible influence can be reduced to a minimum. $\underline{19}$ A strength of the RTD is that it is related to a single-particle problem.

The ITD method has the advantage that tip-molecule interactions can be expected to have, if at all, a marginal influence on the interpeak times (irrespective of tipsubstrate distances or bias voltages). For small interpeak times, the ITD is essentially also related to a singleparticle problem. For large interpeak times, an approximate value of $D$ can be obtained based on an estimate for processes, where one molecule in the detection area under the tip is followed by another molecule. The values obtained in this way turn out to be close to those resulting from the other methods. Compared to the residence times, the interpeak times are quite large and are thus less prone to the experimental time resolution and the threshold value $I_{c}$.

\section{CONCLUSIONS}

In order to exploit the strength of each method one can combine the different methods, if the corresponding data in the relevant time regime have sufficient statistics. For a first classification of the measurement it is helpful to choose the ACF method, because it yields fast results without analyzing the time series in detail. In a subsequent step the peak widths and interpeak intervals should be extracted with the procedure described in Sec. III We then recommend to use the RTD method for determining $D$ whenever the exponential tail is sufficiently pronounced and the tip influence on the diffusion can be neglected. Otherwise the ITD method should be preferred. In any case, one should always apply both the RTD and the ITD method to perform consistency checks and to obtain most reliable results. Our analysis for $\mathrm{CuPc}$ on $\mathrm{Ag}(100)$ shows that, despite diffusion coefficients extracted from the different methods may differ for one or another sample, the activation energies determined from the Arrhenius plots should have comparable values, see Fig. 8 .

An interesting question is whether the methods discussed here can be taken over to other fields. Obviously, the ACF method is well known in fluorescence correla-

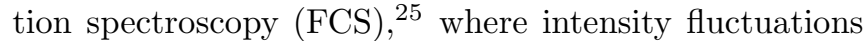
reflect concentration fluctuations, typically in some finite detection volume. Similarly, analysis of density fluctuations has been applied as one variant to determine diffusion coefficients in Field Emission Microscopy $\stackrel{2}{2}$ In the situation discussed in Sec. III only one particle is in the detection area and accordingly information on the tagged particle diffusion (tracer diffusion) is obtained. In the hypothetical limit of very small particle concentrations in FCS, where the mean interparticle distance becomes larger than the linear size of the detection volume, one should essentially recover the behavior discussed in

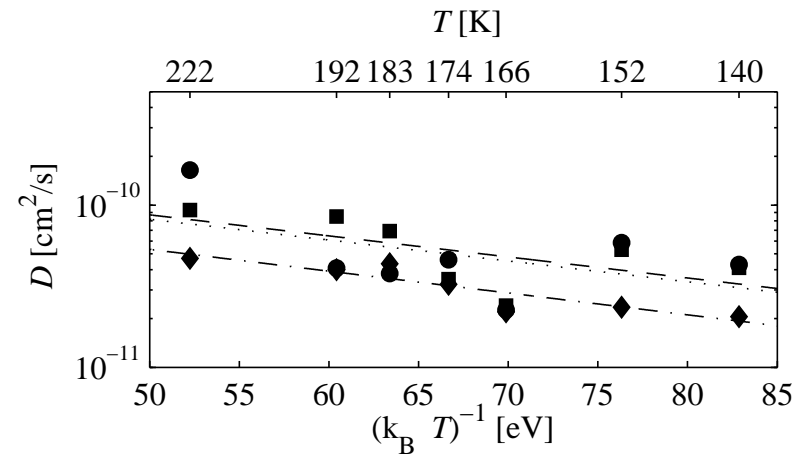

FIG. 8. Comparison of the diffusion coefficients obtained from the three methods. Squares, dashed line: ACF method $\left(E_{a}=30 \mathrm{meV}, D_{0}=10^{-9.4} \mathrm{~cm}^{2} / \mathrm{s}\right)$; Circles, dotted line: RTD method $\left(E_{a}=30 \mathrm{meV}, D_{0}=10^{-9.4} \mathrm{~cm}^{2} / \mathrm{s}\right) ;$ Diamonds, dashed-dotted line: ITD method $\left(E_{a}=31 \mathrm{meV}\right.$, $\left.D_{0}=10^{-9.6} \mathrm{~cm}^{2} / \mathrm{s}\right)$.

Sec. III (with $R$ then playing the role of the detection length). In common applications of this method one is, however, not able to extract the tagged particle information and this makes a difference to the ACF method discussed in Sec. [II] This also prevents the use of the RTD and ITD in the analysis of typical fluorescence signals. These methods can yet become useful in light of the ongoing development of sophisticated techniques to measure single-molecule properties.

In summary, we provided formulas for different means of analyzing fluctuations in the signal of a locally fixed probe on a surface, where the fluctuations are caused by diffusing particles. By exploiting the fact that the molecule extension enters as a scale bar into the equations, a quantitative determination of the diffusion constant becomes possible. So far, we have considered circular shapes for planar molecules. The situation will evidently be more involved when rectangular or even more complicated shapes are considered. In addition rotational diffusion and anisotropies induced by the substrate then can modify the results. As mentioned above, the interaction of the STM tip with the diffusing molecule was neglected so far. The interaction is influenced by the experimental setup (tip shape, tip-substrate distance etc.) and it is specific for the tip and molecule material. One objective could be to minimize the tip influence experimentally. On the other hand, it could be interesting to study how the interaction potential affects the diffusion of the molecule. This potential has so far not been included in the analysis, but it should be possible and bears an interesting route to obtain information on the tip-molecule interaction.

\section{ACKNOWLEDGMENTS}

J.I. and M.S. gratefully acknowledge fincancial support by the SFB 624 of the Deutsche Forschungsgemeinschaft. 


\section{Appendix: Diffusion propagators for RTD and ITD}

The results given in Eqs. (5) and (9) for $p(\mathbf{r}, t)$ have been derived earlier in the literature, in particular Eq. (9) by using the Heaviside method ${ }^{26}$ We give a straightforward derivation here, using separation of variables and eigenfunction expansions.

Let us first consider the equation for diffusion of a particle in a circular stripe $a<r<c$ ( $a$ here not equal to the lattice constant used in the main text) with absorbing boundaries and initial distribution

$$
p_{0}(\mathbf{r})=\frac{1}{2 \pi r} \delta(r-b), a<b<c .
$$

Due to the radial symmetry $p(\mathbf{r}, t)=\rho(r, t) / 2 \pi$, where $\rho(r, t)$ satisfies the radial diffusion equation

$$
\frac{\partial \rho(r, t)}{\partial t}=D\left(\frac{\partial^{2}}{\partial r^{2}}+\frac{1}{r} \frac{\partial}{\partial r}\right) \rho(r, t)
$$

with $\rho(a, t)=\rho(c, t)=0$ and $\rho(r, 0)=\delta(r-b) / r$.

Making the product ansatz $\rho(r, t)=\rho(r, 0) g(t)$, Eq. (A.2) separates in the variables $r$ and $t$. One obtains $g(t)=\mathrm{e}^{-\lambda^{2} D t}$, where the allowed values for $\lambda^{2}$ are the eigenvalues of the radial Laplace operator in the circular stripe,

$$
\left(\frac{\partial^{2}}{\partial r^{2}}+\frac{1}{r} \frac{\partial}{\partial r}\right) \Psi_{n}(r)=-\lambda_{n}^{2} \Psi_{n}(r), \Psi_{n}(a)=\Psi_{n}(c)=0 .
$$

Because the Laplacian is negative definite, $\lambda_{n}^{2}>0$. The eigenfunctions $\Psi_{n}$ are given by linear combinations of the zeroth order Bessel functions $J_{0}($.$) and Y_{0}($.$) of$ first and second kind, $\Psi_{n}(r)=A_{n} J_{0}\left(\lambda_{n} r\right)+B_{n} Y_{0}\left(\lambda_{n} r\right)$ (where restriction to $\lambda_{n}>0$ gives linear independent eigenfunctions). The Dirichlet boundary conditions yield $A_{n} J_{0}\left(\lambda_{n} a\right)=-B_{n} Y_{0}\left(\lambda_{n} a\right)$ [or $A_{n} J_{0}\left(\lambda_{n} c\right)=$ $\left.-B_{n} Y_{0}\left(\lambda_{n} c\right)\right]$, and

$$
J_{0}\left(\lambda_{n} a\right) Y_{0}\left(\lambda_{n} c\right)-J_{0}\left(\lambda_{n} c\right) Y_{0}\left(\lambda_{n} a\right)=0
$$

as determining equation for the $\lambda_{n}, n=1,2, \ldots(0<$ $\left.\lambda_{1}<\lambda_{2}<\ldots\right)$. Introducing the cross product

$$
W(x, y)=J_{0}(x) Y_{0}(y)-J_{0}(y) Y_{0}(x),
$$

the solution becomes

$$
\rho(r, t)=\sum_{n=1}^{\infty} C_{n} W_{0}\left(\lambda_{n} r, \lambda_{n} a\right) \mathrm{e}^{-\lambda_{n}^{2} D t},
$$

where $W_{0}\left(\lambda_{n} a, \lambda_{n} c\right)=0$. Utilizing the orthogonality of the eigenfunctions,

$$
\begin{array}{r}
\int_{a}^{c} \mathrm{~d} r \\
\quad r W_{0}\left(\lambda_{m} r, \lambda_{m} a\right) W_{0}\left(\lambda_{n} r, \lambda_{n} a\right) \\
=\delta_{m n} \int_{a}^{c} \mathrm{~d} r r W_{0}^{2}\left(\lambda_{n} r, \lambda_{n} a\right),
\end{array}
$$

the expansion coefficients $C_{n}$ follow from the initial condition Eq. (A.1):

$$
\begin{aligned}
C_{n} & =\frac{\int_{a}^{c} \mathrm{~d} r r W_{0}\left(\lambda_{n} r, \lambda_{n}, a\right) \rho(r, 0)}{\int_{a}^{c} \mathrm{~d} r r W_{0}^{2}\left(\lambda_{n} r, \lambda_{n} a\right)} \\
& =\frac{W_{0}\left(\lambda_{n} b, \lambda_{n} a\right)}{\int_{a}^{c} \mathrm{~d} r r W_{0}^{2}\left(\lambda_{n} r, \lambda_{n} a\right)} .
\end{aligned}
$$

The result for $p(\mathbf{r}, t)$ thus is

$$
p(\mathbf{r}, t)=\sum_{n=1}^{\infty} \frac{W_{0}\left(\lambda_{n} b, \lambda_{n} a\right) W_{0}\left(\lambda_{n} r, \lambda_{n} a\right)}{2 \pi \int_{a}^{c} \mathrm{~d} r r W_{0}^{2}\left(\lambda_{n} r, \lambda_{n} a\right)} \mathrm{e}^{-\lambda_{n}^{2} D t} .
$$

The diffusion propagator in Eq. (9) relevant for the ITD corresponds to the limit $c \rightarrow \infty$, where the spectrum of eigenvalues determined by Eq. (A.4) becomes continuous. Analogous to the change of a Fourier series to a Fourier integral, we are led to consider the Weber transform ${ }^{27}$ of a function $f=f(r)$

$$
F(\lambda)=\frac{1}{a^{2}} \int_{a}^{\infty} \mathrm{d} r r W_{0}(\lambda r, \lambda a) f(r),
$$

with back-transformation

$$
f(r)=a^{2} \int_{0}^{\infty} \mathrm{d} \lambda \lambda \frac{W_{0}(\lambda r, \lambda a)}{J_{0}^{2}(\lambda a)+Y_{0}^{2}(\lambda a)} F(\lambda) .
$$

Accordingly, Eq. (A.6) becomes

$$
\rho(r, t)=\int_{0}^{\infty} \mathrm{d} \lambda C(\lambda) W_{0}(\lambda r, \lambda a) \mathrm{e}^{-\lambda^{2} D t},
$$

where

$$
\begin{aligned}
C(\lambda) & =\frac{\lambda}{J_{0}^{2}(\lambda a)+Y_{0}^{2}(\lambda a)} \int_{a}^{\infty} \mathrm{d} r r W_{0}(\lambda r, \lambda a) \rho(r, 0) \\
& =\frac{\lambda W_{0}(\lambda b, \lambda a)}{J_{0}^{2}(\lambda a)+Y_{0}^{2}(\lambda a)} .
\end{aligned}
$$

This yields

$$
p(\mathbf{r}, t)=\int_{0}^{\infty} \frac{\mathrm{d} \lambda}{2 \pi} \lambda \frac{W_{0}(\lambda r, \lambda a) W_{0}(\lambda b, \lambda a)}{J_{0}^{2}(\lambda a)+Y_{0}^{2}(\lambda a)} \mathrm{e}^{-\lambda^{2} D t} .
$$

Equation (9) follows by setting $a=R, b=(1+\epsilon) R$, and $\lambda=\alpha / R$.

For the diffusion propagator in Eq. (5) relevant for the RTD only one boundary condition $\rho(c, t)=0$ has to be taken into account. In this case the Bessel functions of second kind cease to apply, because their logarithmic singularity at the origin eliminates them from the space of functions, where the radial Laplace operator is Hermitean. Notice that the logarithmic singularity would be no problem with respect of the integrability of $p(\mathbf{r}, t)$. The eigenfunctions thus are given by $\Psi_{n}(r)=A_{n} J_{0}\left(\lambda_{n} r\right)$, where the $\lambda_{n}$ are determined by $J_{0}\left(\lambda_{n} c\right)=0$. Equation (A.6) becomes

$$
\rho(r, t)=\sum_{n=1}^{\infty} A_{n} J_{0}\left(\lambda_{n} r\right) \mathrm{e}^{-\lambda_{n}^{2} D t},
$$


and the $A_{n}$ are again determined by the initial condition, corresponding to an expansion of $\rho(r, 0)$ into a FourierBessel series,

$$
A_{n}=\frac{\int_{0}^{c} \mathrm{~d} r r J_{0}\left(\lambda_{n} r\right) \rho(r, 0)}{\int_{0}^{c} \mathrm{~d} r r J_{0}^{2}\left(\lambda_{n} r\right)}=\frac{2 J_{0}\left(\lambda_{n} b\right)}{c^{2} J_{1}^{2}\left(\lambda_{n} c\right)} .
$$

This yields

$$
p(\mathbf{r}, t)=\frac{1}{\pi c^{2}} \sum_{n=1}^{\infty} \frac{J_{0}\left(\lambda_{n} r\right) J_{0}\left(\lambda_{n} b\right)}{J_{1}^{2}\left(\lambda_{n} c\right)} \mathrm{e}^{-\lambda_{n}^{2} D t} .
$$

Equation (5) follows by setting $c=R, b=(1-\epsilon) R$ and $\lambda_{n}=\alpha_{n} / R$.
* philipp.maass@uni-osnabrueck.de

1 E. W. Mülller, Zeits. f. Physik 108, 668 (1938).

2 R. Gomer, Rep. Prog. Phys. 53, 917 (1990).

3 E. W. Müller and T. T. Tsong, Field Ion Microscopy (American Elsevier Publishing Company, 1969).

4 R. Viswanathan, D. Burgess, P. Stair, and E. Weitz, J. Vac. Sci Technol. 20, 605 (1982).

5 S. George, A. Desantolo, and R. Hall, Surf. Sci. 159, L425 (1985).

${ }^{6}$ E. Ganz, S. K. Theiss, I.-S. Hwang, and J. Golovchenko, Phys. Rev. Lett. 68, 1567 (1992).

7 R. Gomer, Appl. Phys. A 39, 1 (1986).

8 B. S. Swartzentruber, Phys. Rev. Lett. 76, 459 (1996).

9 J. V. Barth, Surf. Sci. Rep. 40, 75 (2000).

${ }^{10}$ H. Brune, Surf. Sci. Rep. 31, 125 (1998).

11 J. W. Evans, P. A. Thiel, and M. C. Bartelt, Surf. Sci. Rep. 61, 1 (2006).

12 M. Einax, S. Ziehm, W. Dieterich, and P. Maass, Phys. Rev. Lett. 99, 016106 (2007).

13 M. Einax, W. Dieterich, and P. Maass, J. Appl. Phys. 105, 054312 (2009).

14 A. Kühnle, Curr. Opin. Colloid Interface Sci. 14, 157 (2009).

15 M. L. Lozano and M. C. Tringides, Europhys. Lett. 30,
$537(1995)$.

16 M. Sumetskii and A. A. Kornyshev, Phys. Rev. B 48, 17493 (1993).

17 M. Sumetskii, A. A. Kornyshev, and U. Stimming, Surf. Sci. 307-309, 23 (1994).

18 M. C. Tringides and M. Hupalo, J. Phys. Condens. Matter 22, 264002 (2010).

19 J. Ikonomov, P. Bach, R. Merkel, and M. Sokolowski, Phys. Rev. B 81, 161412(R) (2010).

20 J. Tersoff and D. R. Hamann, Phys. Rev. Lett. 50, 1998 (1983).

21 A. Mugarza, R. Robles, C. Krull, R. Korytár, N. Lorente, and P. Gambardella, Phys. Rev. B 85, 155437 (2012)

${ }^{22}$ P. Bach, diploma thesis, University of Bonn (2009).

23 The offset corresponding to the shift is associated with the preset setpoint current that is mainly related to tunneling to/from the bare Ag surface.

24 S. Redner, A guide to first-passage processes (Cambride University Press, 2001).

25 E. P. Petrov and P. Schwille, State of the Art and Novel Trends in Fluorescence Correlation Spectroscopy (Springer, 2008) pp. 145-197.

26 S. Goldstein, Proc. London Math. Soc. 34, 51 (1932).

27 B. Davies, Integral Transforms and Their Applications (Springer, 1978). 